\title{
Stroke in surgery of the thoracic aorta: Incidence, impact, etiology, and prevention
}

Lee J. Goldstein, MD

Ryan R. Davies, MD

John A. Rizzo, PhD

Javier J. Davila, MD

Matthew R. Cooperberg, MD, MPH

Richard K. Shaw, MD

Gary S. Kopf, MD

John A. Elefteriades, MD
From the Department of Surgery, Section of Cardiothoracic Surgery, and Yale University School of Medicine, New Haven, Conn.

Received for publication June 21, 2000; revisions requested Sept 14, 2000; revisions received April 5, 2001; accepted for publication May 2, 2001.

Address for reprints: John A. Elefteriades, MD, Section of Cardiothoracic Surgery, Yale University School of Medicine, 333 Cedar St, FMB 121, New Haven, CT 06510 (Email: john.elefteriades@yale.edu).

J Thorac Cardiovasc Surg 2001;122:935-45

Copyright (C) 2001 by The American Association for Thoracic Surgery

0022-5223/2001 $\$ 35.00+0 \quad \mathbf{1 2 / 1 / 1 1 7 2 7 6}$

doi:10.1067/mtc.2001.117276
Objectives: To determine the incidence, impact, etiology, and methods for prevention of stroke after surgery of the thoracic aorta.

Methods: A total of 317 thoracic aortic operations on 303 patients (194 male, 109 female) aged 13 to 87 years (mean 61 years) were reviewed. There were 218 procedures on the ascending aorta and arch and 99 on the descending aorta. Of the 218 procedures on the ascending aorta and arch, 86 involved cardiopulmonary bypass, 122 involved deep hypothermic circulatory arrest, 2 involved antegrade cerebral perfusion, and 8 involved "clamp and sew" or left heart bypass. Of the 99 procedures on the descending aorta, 20 involved "clamp and sew," 69 involved left heart or full bypass, and 10 involved deep hypothermic circulatory arrest. A total of 206 cases were elective and 97 were emergency operations.

Results: Twenty-three (7.3\%) of 317 patients had a stroke. Fifteen strokes occurred in operations on the ascending aorta and 8 in operations on the descending aorta (6.9\% vs $8.1 \% ; P=.703)$. Stroke occurred in $16(16.5 \%)$ of 97 emergency operations and $7(3.4 \%)$ of 206 elective operations $(P=.001)$. In the 300 patients surviving the operation, stroke was a significant predictor of postoperative death $(9 / 23$ [39.1\%] vs $23 / 277$ [8.3\%]; $P=.001$ ). Analysis of operative reports, brain images, and neurologic consultations revealed 15 of the 23 strokes were embolic, 3 were ischemic, 3 hemorrhagic, and 2 indeterminate. Patients with stroke had longer intensive care unit stays (18.4 vs 6.8 days; $P=.0001$ ), longer times to extubation ( 12.7 vs 3.8 days; $P<.0012$ ), longer postoperative stays (31.4 vs 14.3 days; $P=.001$ ), and decreased age-adjusted survival (relative risk 2.775; $P=.0013$ ). After implementation of a rigorous antiembolic regimen, both strokes and mortality trended downward.

Conclusions: (1) Stroke complicates surgery of both the ascending and descending thoracic aorta and warrants consideration in decision making. (2) Strokes are largely embolic. (3) Antiembolic measures for particles and air are essential, including gentle aortic manipulation, thorough debridement, transesophageal echocardiography to identify aortic atheromas, carbon dioxide flooding of the field, and (in descending cases) proximal clamp application before initiating femoral perfusion.

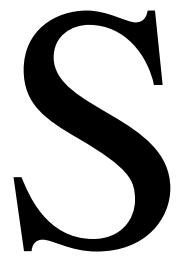

troke is a devastating complication of surgery of the thoracic aorta. The surgeon must balance the life-threatening sequelae of untreated thoracic aortic disease with the risks associated with surgical repair. Surgeons have often focused on death, bleeding, and paraplegia as the major adverse outcomes of thoracic aortic surgery. This study aims to scrutinize specifically the complication of stroke after aortic surgery. 
TABLE 1. Demographic and clinical characteristics

\begin{tabular}{lc}
\hline Characteristic & Incidence \\
\hline Tobacco use & $102(32.2 \%)$ \\
Ethanol use & $24(7.6 \%)$ \\
Prior CVA & $35(11.0 \%)$ \\
COPD & $30(9.5 \%)$ \\
CAD & $70(22.1 \%)$ \\
Obesity & $19(6.0 \%)$ \\
Valvular heart disease & $124(39.1 \%)$ \\
CHF & $32(10.1 \%)$ \\
Prior vascular surgery & $13(4.1 \%)$ \\
Prior AAA & $20(6.3 \%)$ \\
Prior aortic surgery & $39(12.3 \%)$ \\
Prior cardiac surgery & $29(9.1 \%)$ \\
Chronic renal failure & $18(5.7 \%)$ \\
Hypertension & $179(56.5 \%)$ \\
Peripheral vascular disease & $19(6.0 \%)$ \\
Hypercholesterolemia & $34(10.7 \%)$ \\
Diabetes & $13(4.1 \%)$
\end{tabular}

$A A A$, Abdominal aortic aneurysm; $C A D$, coronary artery disease; $C H F$, congestive heart failure; $C O P D$, chronic obstructive pulmonary disease; $C V A$, cerebrovascular accident.

Most clinical reports on aortic surgical procedures simply state an overall incidence of stroke without specific analysis of this complication. ${ }^{1-4}$ Stroke may not be adequately appreciated as a common sequel of descending aortic operations. Additionally, even regarding the ascending aorta, it is often difficult to compare interinstitutional data because of differences in the use of deep hypothermic circulatory arrest, retrograde cerebral perfusion, and antegrade cerebral perfusion during the arrest interval. Our study focuses specifically on the complication of stroke and the specific mechanisms by which the strokes in our patients occurred. The objectives of this study are as follows: (1) to determine the incidence of stroke as a complication of aortic surgery on the basis of data from Yale-New Haven Hospital, (2) to determine the clinical impact these strokes have on early and late survival, (3) to analyze the clinical details of those patients having strokes to determine the causes of these strokes, (4) to give suggestions for prevention of strokes on the basis of the pathologic mechanisms by which they occur, and (5) to analyze the effectiveness of those recommendations.

\section{Methods}

Data collection for this study occurred in two steps. In 1999, we retrospectively analyzed 227 consecutive thoracic aortic operations performed on 222 patients from January 1989 through September 1998. All surgical procedures for thoracic aortic disease (aneurysms, dissections, penetrating atherosclerotic ulcers, or intramural hematomas) were included. Reimplantation of the coronary arteries, concomitant coronary revascularization procedures, and resuspension or replacement of the aortic valve were per-
TABLE 2. Operative details

\begin{tabular}{lc}
\hline No. of operations & 317 \\
Age (y) & $13-87$ \\
Range & 61 \\
Mean & $194(64.0 \%)$ \\
Sex & $109(36.0 \%)$ \\
Male & \\
Female & $97(32.0 \%)$ \\
Type of operation & $55(17.4 \%)$ \\
Emergency operation & \\
Aortic rupture & $78(24.6 \%)$ \\
Pharmacologic treatment & $87(27.4 \%)$ \\
Aminocaproic acid & $45(14.2 \%)$ \\
Aprotinin & $175(55.2 \%)$ \\
Barbiturates & \\
Steroids & \\
Location & 8 \\
Ascending aorta/arch (n = 218)* & \\
Clamp and sew or left heart bypass $\dagger$ & 86 \\
CPB alone & 2 \\
Selective antegrade perfusion + CPB & 122 \\
DHCA + CPB & \\
Descending aorta (n = 99) $\ddagger$ & 20 \\
Clamp and sew & 69 \\
CPB (left heart or full) & 10 \\
DHCA &
\end{tabular}

$\overline{C P B}$, Cardiopulmonary bypass; DHCA, deep hypothermic circulatory arrest.

*Mean arrest time was 33.4 minutes at $17.7^{\circ} \mathrm{C}$.

tUnusual cases (eg, base of innominate artery injury or distal arch operation). $\ddagger$ Mean arrest time was 40.9 minutes at $16.2^{\circ} \mathrm{C}$.

formed as indicated. Cerebrospinal fluid drainage was routinely used in descending aortic operations. After analyzing these patients, we instituted specific antiembolic preventive measures, as described fully in the "Results" section. These measures included gentle aortic manipulation, thorough debridement, transesophageal echocardiography to identify aortic atheromas, flooding of the field with carbon dioxide, proximal clamp application before initiating femoral perfusion (in operations on the descending aorta), and avoidance of left atrial cannulation in cases of atrial fibrillation. Subsequent to these changes, between October 1998 and December 2000, we collected another 90 procedures on 84 patients. This second group is compared with the first to assess the impact of these antiembolic measures.

Charts were reviewed and data points collected as listed in Table 1. Our review of 317 operations yielded 303 patients, 194 of whom were male $(64.0 \%)$ and 109 female (36.0\%). Patient age ranged from 13 to 87 years, with a mean age of 61 years. Operative details are summarized in Table 2. No retrograde cerebral perfusion was used in our study. Carbon dioxide flooding was used uniformly in all operations and cerebrospinal fluid drainage was used for operations on the descending aorta. A total of $206(68.0 \%)$ operations were elective and $97(32.0 \%)$ were emergency procedures, with 55 aortic ruptures, defined as free blood in the pericardium or mediastinum. Long-term follow-up was conducted 
TABLE 3. Stroke rates

\begin{tabular}{|c|c|c|c|c|c|}
\hline & Overall $(n=317)$ & Ascending ( $n=218$ ) & Descending ( $n=99$ ) & Elective $(\mathrm{n}=206)^{*}$ & Emergency $(\mathrm{n}=97)^{*}$ \\
\hline Overall & $23(7.3 \%)$ & $15(6.9 \%)$ & $8(8.1 \%)$ & $7(3.4 \%)$ & $16(16.5 \%)$ \\
\hline & & \multicolumn{2}{|c|}{$P=.703$} & \multicolumn{2}{|c|}{$P=.001$} \\
\hline
\end{tabular}

*The number of elective and emergency cases does not equal the total of 317 cases because inadequate data were available to accurately classify 14 procedures.

TABLE 4. Risk factors for stroke- univariate analysis $\left(\chi^{2}\right.$ test) of preoperative risk factors

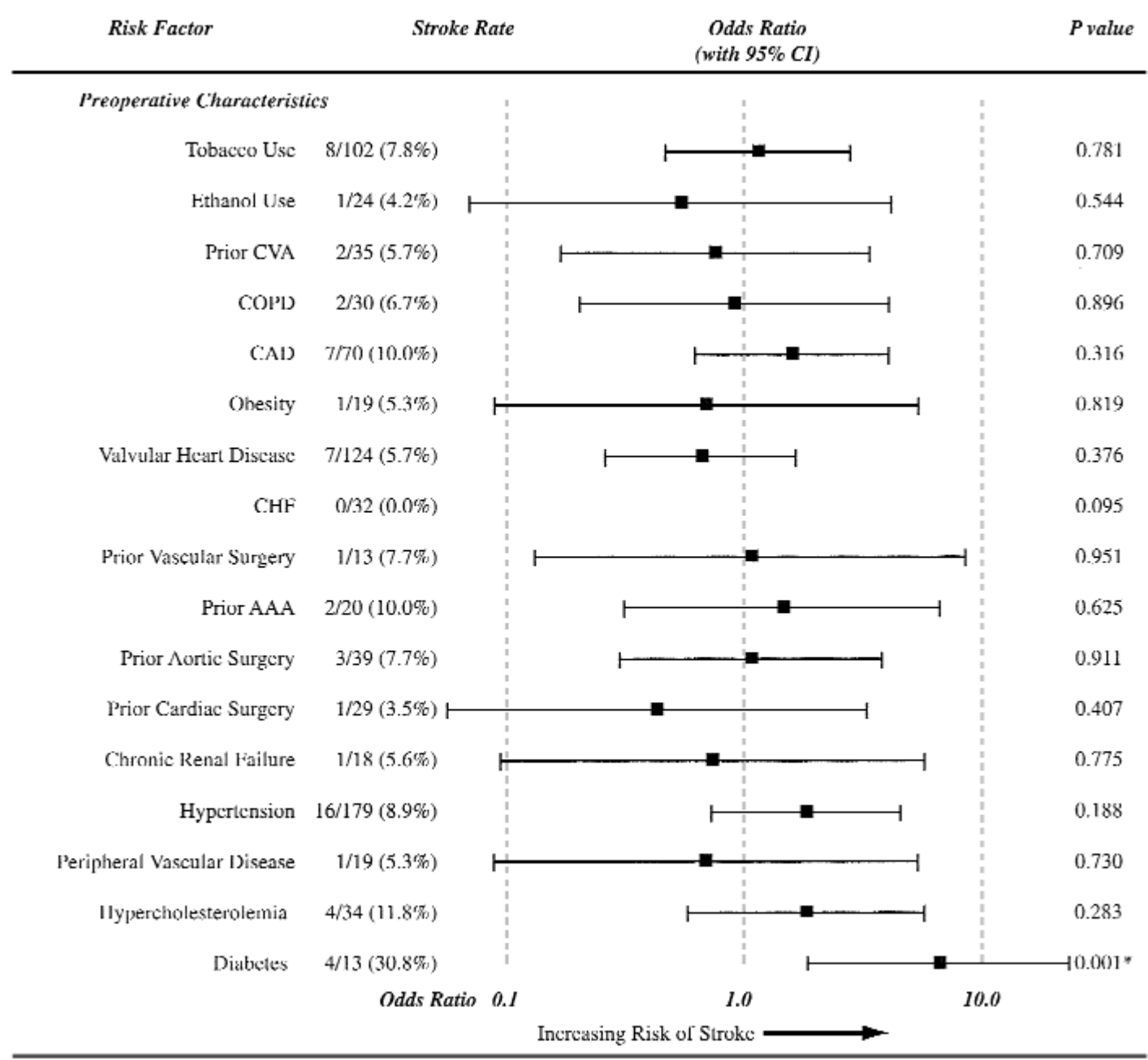

Bars on graph indicate $95 \%$ confidence intervals; odds ratios cannot be calculated when the incidence of disease is zero. $C l$, Confidence intervals; $C V A$, cerebrovascular accident; $C O P D$, chronic obstructive lung disease; $C A D$, coronary artery disease; $C H F$, congestive heart failure; $A A A$, abdominal aortic aneurysm; $S C A$, subclavian artery; $I A B P$, intra-aortic balloon pump.

${ }^{*}$ Statistically significant result.

through office visits, telephone interviews, and analysis of office records. In this review, we used operative reports, computed tomographic scans, magnetic resonance imaging, and neurologic consultation reports in combination with neuroradiologic consultation to determine the causes of these strokes.

Univariate and multivariate logistic regression analysis was performed to investigate preoperative and intraoperative predictors of stroke by means of the logistic regression procedure of the SAS System (SAS Institute, Inc, Cary, NC). Preoperative risk factors were stratified according to previously published standards. ${ }^{5}$ Univariate analysis of number of days intubated, intensive care unit length of stay, and postoperative length of stay was performed to determine significant differences in clinical outcomes of patients who had a stroke. The Student $t$ test was employed for univariate 
TABLE 5. Risk factors for stroke-univariate analysis $\left(\chi^{2}\right.$ test) of operative risk factors

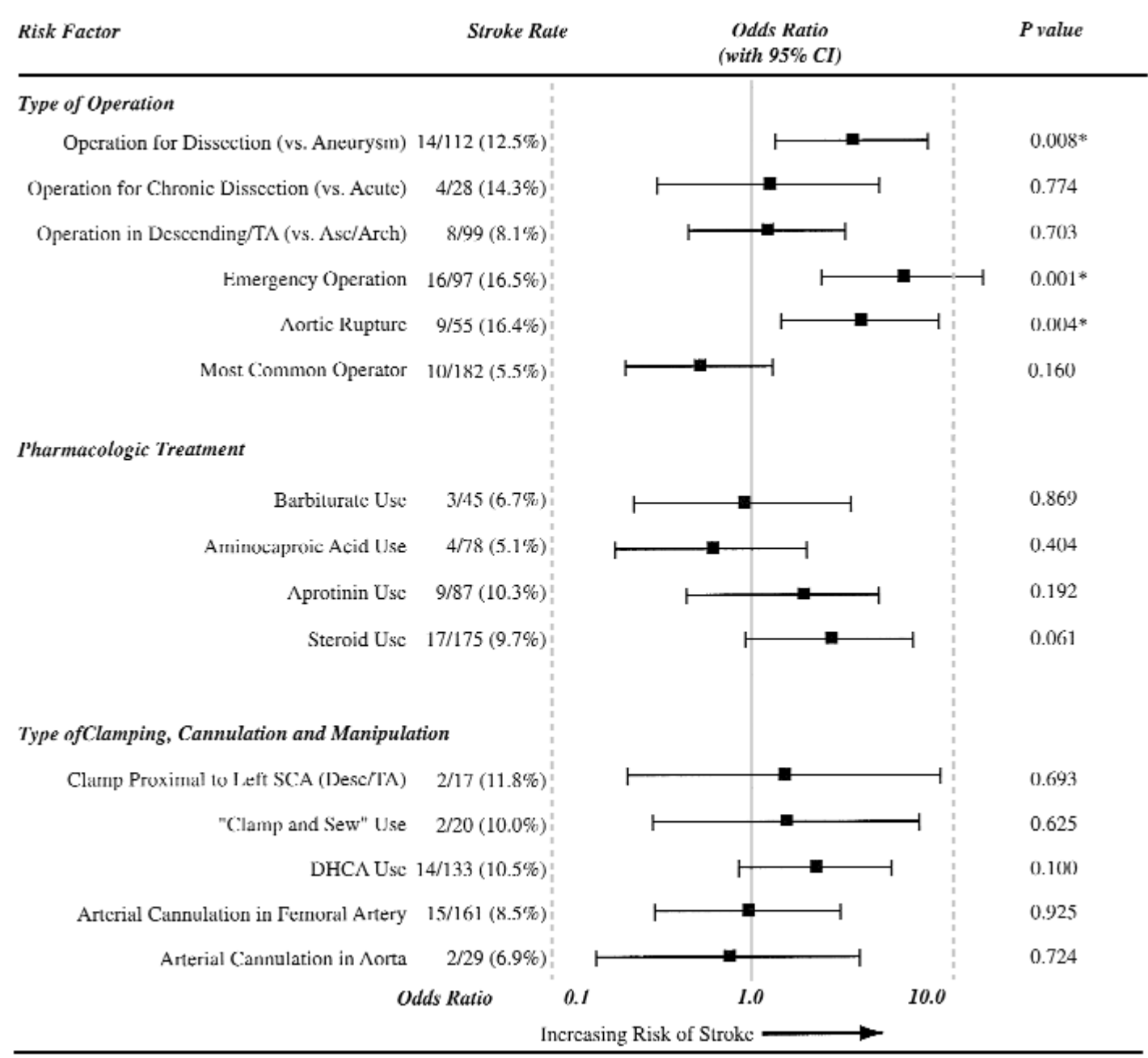

Bars on graph indicate $95 \%$ confidence intervals; odds ratios cannot be calculated when the incidence of disease is zero. $\mathrm{Cl}$, confidence intervals; $\mathrm{CVA}$, cerebrovascular accident; $C O P D$, chronic obstructive lung disease; $C A D$, coronary artery disease; $C H F$, congestive heart failure; $A A A$, abdominal aortic aneurysm; $S C A$, subclavian artery; IABP, intra-aortic balloon pump; $D H C A$, deep hypothermic circulatory arrest; $A s c$, ascending; $T A$, thoracoabdominal.

${ }^{*}$ Statistically significant result.

analysis with the use of the analysis of variance procedure of the SAS System. Kaplan-Meier survival analysis was performed and the log-rank calculated; results were also adjusted for age by means of proportional hazards regression, both with the SAS System.

\section{Results}

\section{Stroke Incidence}

Overall, 23 (7.3\%) of 317 operations resulted in cerebrovascular accident, defined as a permanent, new, central neurologic deficit. Spinal cord ischemia was not included in this analysis, because our intent was to focus on discrete lesions of the brain. The incidence of stroke is compared for location of aortic disease and urgency of procedure in Table 3. Tables 4,5 , and 6 illustrate univariate analyses of risk factors predictive of stroke in this population.
In a univariate analysis, preoperative characteristics had little impact on the incidence of stroke (Table 4). Stroke occurred at similar rates in operations on the ascending $(6.9 \%)$ and descending $(8.1 \%)$ aorta (Table 3$)$. On the other hand, patients requiring emergency aortic surgery had significantly higher rates of stroke (odds ratio 5.62, 95\% confidence intervals 2.23-14.16) than those undergoing elective operations (Tables 3 and 5). Other operative characteristics associated with higher stroke rates included operations for dissection and those for aortic rupture (Table 5). Although the use of deep hypothermic circulatory arrest had no impact on stroke rates, longer arrest times were associated with increased stroke rates (Table 6).

Multivariate logistic regression analysis revealed some additional factors (both preoperative and intraoperative) that 


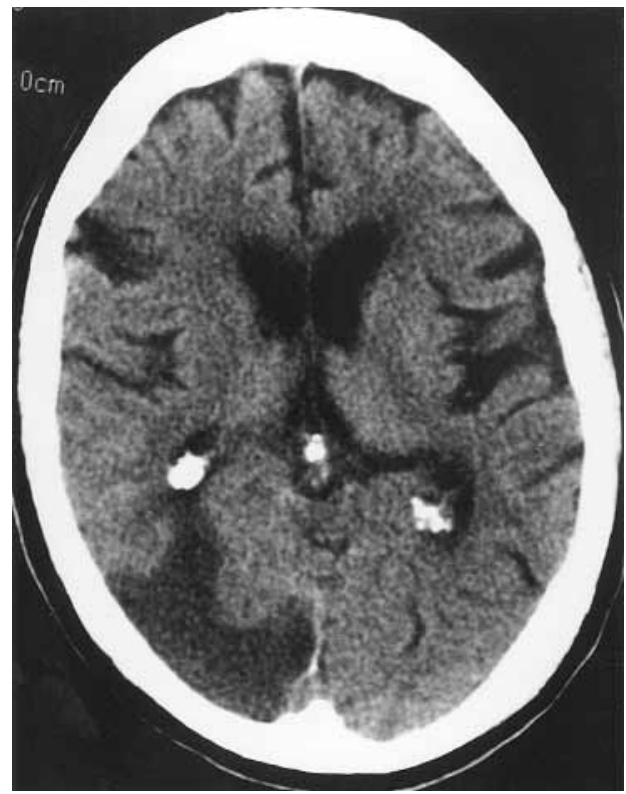

Figure 1. Computed tomographic scan of embolic stroke. Note the hypodense infarct in the right occipital region, with marked loss of brain substance. There is no midline shift.

increased a patient's risk of stroke during aortic surgery; these included surgery in the descending aorta, emergency surgery, the use of deep hypothermic circulatory arrest, increased use of fresh frozen plasma, and a history of diabetes mellitus (Table 7). None of the other variables from the univariate analysis met the threshold $(P<.30)$ for entry into the regression model. In addition, there was no significant association between the location of the proximal clamp in descending aortic surgery or the arterial cannulation site and the incidence of stroke.

The surgeon performing the procedure had no statistically significant effect on stroke incidence but did influence the mortality of the procedure $(P=.001)$ (Table 8$)$. Overall 30-day mortality was $49(15.5 \%)$ deaths in 317 operations, but for the surgeon who performed the most procedures (182), the 30-day mortality rate was only $8.8 \%(P=.001)$. Mortality also varied in these patients on the basis of the urgency of the operation. Elective operations had a 7.3\% (15/191) 30-day mortality versus a $34.0 \%$ (33/97) 30-day mortality for emergency operations $(P=.0001)$. Mortality was higher in patients with descending operations than in those with either ascending or thoracoabdominal operations (Table 9), reflecting the relatively large number of ruptured aortas in the descending category $(15 / 63,23.8 \%)$.

\section{Etiology of Stroke}

Using operative reports (specifically, surgeons' reports of severe atheroma or clot), neurologic clinical consultation, and neuroradiologic evaluation of stroke pattern on computed tomography or magnetic resonance imaging, we were

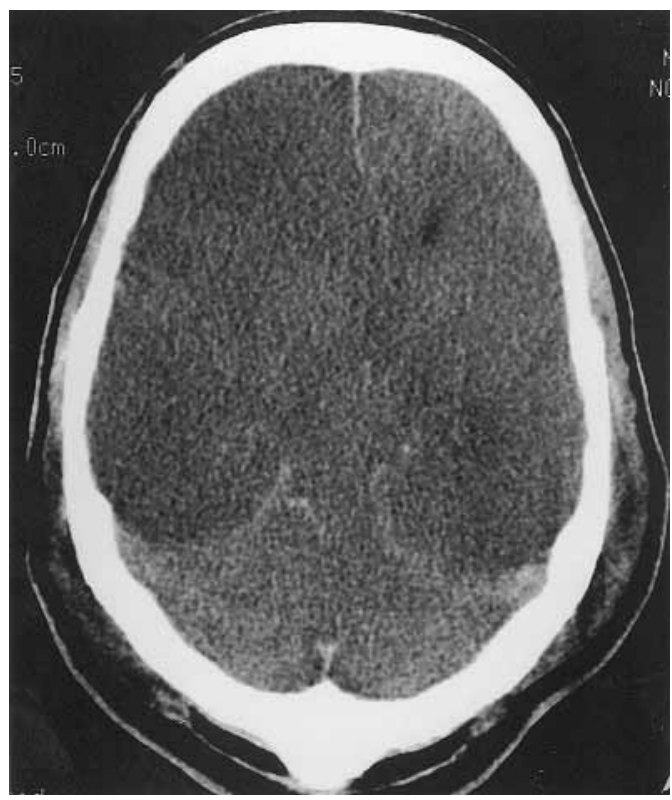

Figure 2. Computed tomographic scan of ischemic stroke. Note the diffuse edema of the entire brain, with obliteration of all cisterns.

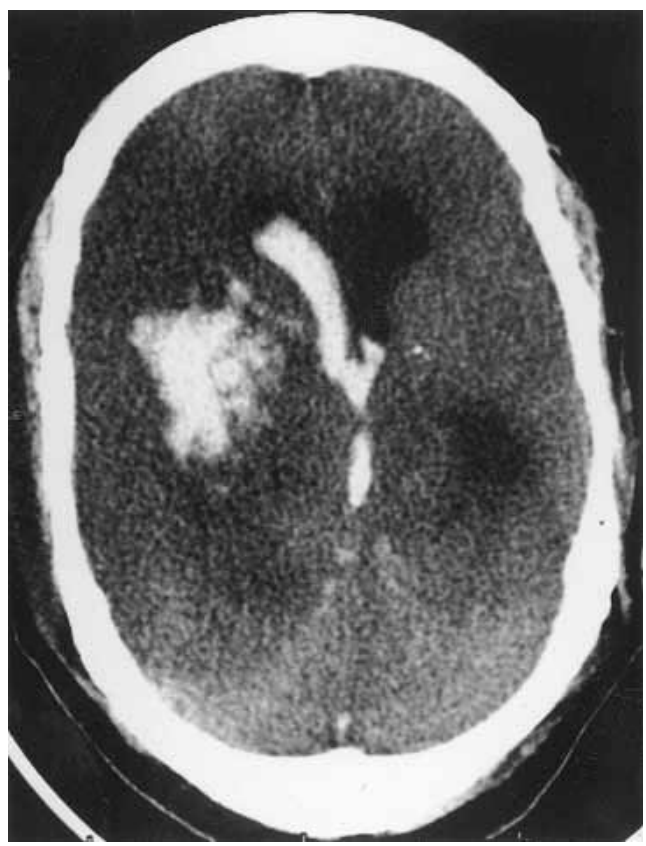

Figure 3. Computed tomographic scan of hemorrhagic stroke. Note the obvious extravasation of blood and midline shift resulting from the mass effect of the bleeding.

able to assign a specific cause for all except 2 of the strokes. Of the strokes encountered, $65.2 \%(15 / 23)$ were embolic in origin, $13.0 \%$ (3/23) were ischemic, and $13.0 \%$ (3/23) were hemorrhagic. The cause of 2 patients' strokes could not be 


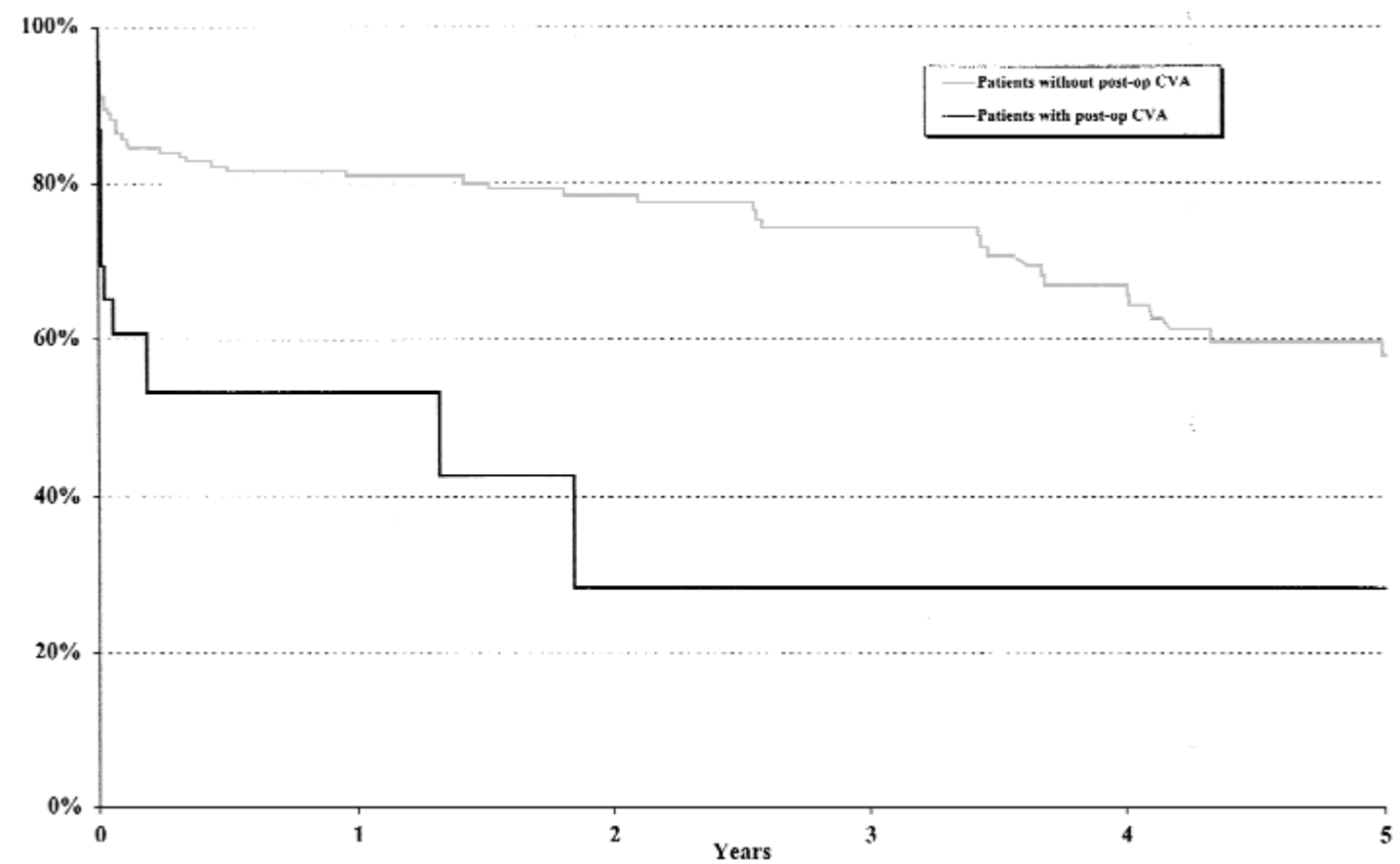

Figure 4. Kaplan-Meier survival analysis of patients having a stroke after aortic surgery. CVA, Cerebrovascular accident.

TABLE 6. Risk factors for stroke-univariate analysis (1-way analysis of variance tests)

\begin{tabular}{|c|c|c|c|}
\hline \multirow[b]{2}{*}{ Risk factor } & \multicolumn{2}{|c|}{ Mean value } & \multirow[b]{2}{*}{$P$ value } \\
\hline & CVA & No CVA & \\
\hline Operative date & $1 / 4 / 96$ & $5 / 5 / 96$ & .642 \\
\hline Anesthesia time (min) & 454.4 & 420.0 & .2774 \\
\hline Operative time (min) & 363.8 & 336.0 & .4701 \\
\hline CPB time $(\min )^{*}$ & 159.3 & 144.2 & .3412 \\
\hline Aortic crossclamping (min) ${ }^{*}$ & 94.7 & 88.0 & .5015 \\
\hline DHCA time (min) $\dagger$ & 37.3 & 31.0 & $.0279 \ddagger$ \\
\hline Lowest core temperature $\left({ }^{\circ} \mathrm{C}\right) \dagger$ & 17.9 & 17.1 & .4533 \\
\hline Crystalloids (intraoperative) (mL) & 4352 & 3549 & .2295 \\
\hline Colloids (intraoperative) (mL) & 50.0 & 214.3 & .3341 \\
\hline FFP (intraoperative) (units) & 3.91 & 1.61 & $.0003 \ddagger$ \\
\hline pRBCs (intraoperative) (units) & 4.21 & 2.67 & .096 \\
\hline Platelets (intraoperative) (units) & 5.83 & 2.76 & $.0017 \dagger$ \\
\hline Cell salvage (intraoperative) (units) & 4.83 & 3.89 & .7308 \\
\hline Urine output (intraoperative) (mL) & 1317 & 1118 & .286 \\
\hline Preoperative heart rate & 80.9 & 77.3 & .3035 \\
\hline Postoperative heart rate & 92.0 & 93.0 & .9183 \\
\hline Preoperative MAP & 82.9 & 90.7 & .3325 \\
\hline Postoperative MAP & 54.9 & 55.9 & .924 \\
\hline Postoperative PAD & 16.8 & 15.7 & .5718 \\
\hline Postoperative $\mathrm{Cl}$ & 2.95 & 2.64 & .3784 \\
\hline
\end{tabular}

$\mathrm{Cl}$, Cardiac index; $C P B$, cardiopulmonary bypass; CVA, cerebrovascular accident; $D H C A$, deep hypothermic circulatory arrest; FFP, fresh frozen plasma; $M A P$, mean arterial pressure; $P A D$, pulmonary artery diastolic pressure; $p R B C s$, packed red blood cells.

${ }^{*}$ Analysis performed only for those patients where relevant time (CPB or crossclamp) was not zero.

tAnalysis performed only for those patients undergoing DHCA.

$\ddagger$ Statistically significant result. determined. Preoperative and intraoperative data and detailed outcomes for patients with stroke are presented in Table 10. Neuroradiologic representations of embolic, ischemic, and hemorrhagic strokes are illustrated in Figures 1 to 3 .

\section{Clinical Impact}

Stroke was a significant predictor of postoperative mortality. Of the 300 patients surviving the operation, 9 (39.1\%) of 23 patients with stroke died during the postoperative hospital stay, compared with $23(8.3 \%)$ of 277 patients without stroke $(P=.001)$. Patients with stroke accounted for $9(28.1 \%)$ of the 32 postoperative deaths. Of those surviving the postoperative hospital stay, patients having a stroke stayed an average of 11.6 days longer in the intensive care unit than those without a stroke (18.4 vs 6.8 days; $P=.0001)$. Additionally, patients with a stroke remained intubated an average of 8.9 days longer than those without stroke (12.7 vs 3.8 days; $P=$ .0012). Finally, patients with a stroke stayed an average of 17.1 days longer in the hospital after the operation than those without (31.4 vs 14.3 days; $P=.001$ ).

Long-term follow-up of patients via office visits, telephone calls, and office records shows a marked difference in survival, with those patients who had a stroke exhibiting a greatly decreased long-term survival compared with those without a stroke $(P=.0001)$ (Figure 4$)$. This difference persists when the results are adjusted for patient age by means of proportional hazards regression (relative risk $=2.775$, $P=.0013)$. 
TABLE 7. Logistic regression analysis of factors predicting rupture or acute dissection (dependent variables)*

\begin{tabular}{|c|c|c|c|c|c|}
\hline Variable & Parameter estimate & SE & $P$ value & OR & $(95 \%$ Cl) \\
\hline Intercept term† & -4.6535 & 0.6719 & .0001 & & \\
\hline Desc/TA surgery & 0.8325 & 0.575 & .1477 & 2.299 & $(0.745-7.095)$ \\
\hline Emergency surgerył & $1.6069 \ddagger$ & 0.5117 & $.0017 \ddagger$ & $4.987 \ddagger$ & $(1.829-13.596)$ \\
\hline History of DM¥ & $2.0097 \ddagger$ & 0.7324 & $.0061 \ddagger$ & $7.461 \ddagger$ & $(1.776-31.350)$ \\
\hline Use of DHCA§ & $1.2457 \S$ & 0.0122 & $.0262 \S$ & $3.475 \S$ & $(1.159-10.424)$ \\
\hline FFP (risk/unit)§ & $0.1143 \S$ & 0.0534 & $.0322 \S$ & $1.121 \S$ & $(1.010-1.245)$ \\
\hline
\end{tabular}

$C l$, Confidence intervals; Desc, descending; $D H C A$, deep hypothermic circulatory arrest; $D M$, diabetes mellitus; $F F P$, fresh frozen plasma; $O R$, odds ratio; $S E$, standard error; $T A$, thoracoabdominal.

*This variable equals 1 if the patient incurred a stroke and 0 otherwise. Forward selection with entry at $P<.030$.

tCriteria for assessing model fit: -2 log L: intercept only: 161.526; intercept and covariates: $131.235 ; \chi^{2}$ for covariates: 30.291 with 5 degrees of freedom $(P=.0001)$.

¥Statistically significant at $P<.01$ level.

§Statistically significant at $P<.05$ level.

TABLE 8. Complications by surgeon

\begin{tabular}{lccc}
\hline Surgeon & $\begin{array}{c}\text { Procedures } \\
\text { performed }\end{array}$ & $\begin{array}{c}\text { Stroke rate } \\
(\%)\end{array}$ & $\begin{array}{c}\text { Mortality } \\
\text { rate (\%) }\end{array}$ \\
\hline A & 182 & 5.5 & 8.8 \\
B & 30 & 10.0 & 30.0 \\
C & 19 & 10.5 & 15.8 \\
D & 18 & 5.6 & 11.1 \\
E & 18 & 5.6 & 22.2 \\
F & 17 & 17.7 & 17.7 \\
G & 12 & 0.0 & 25.0 \\
H & 7 & 14.3 & 57.1 \\
Others & $1.75^{*}$ & 14.3 & 35.7 \\
Pvalue & & .554 & .001 \\
\hline
\end{tabular}

${ }^{*}$ Average number of procedures performed per surgeon included in this group.

This clinical information indicated the importance of embolism in the origin of stroke in these patients. A program of antiembolic measures was systematically instituted at our center thereafter, corresponding to operations performed during and after November 1998. These measures included use of intraoperative transesophageal echocardiography to identify aortic atheroma, thorough debridement of atheroma, use of the subclavian artery for cannulation (rather than the femoral) in cases of severe arteriosclerosis of the descending aorta, carbon dioxide flooding of the field, avoidance of the left atrium for cannulation in cases of atrial fibrillation, and routine clamping of the proximal thoracic aorta before starting left atrial-femoral artery bypass. Carbon dioxide flooding of the field was used throughout the entire experience, before and after the vigorous antiembolic regimen.

Since the institution of these changes, the overall stroke rate has decreased (from $7.9 \%$ to $5.6 \% ; P=.463$ ). This decrease is most pronounced in surgery on the descending aorta, where the stroke rate has decreased from 8 in 77 cases
TABLE 9. Mortality rates

\begin{tabular}{lcccc}
\hline Procedure & $\begin{array}{c}\text { No. } \\
\text { performed }\end{array}$ & $\begin{array}{c}\text { Intraop } \\
\text { death }\end{array}$ & $\begin{array}{c}\text { Postop } \\
\text { deaths }\end{array}$ & $\begin{array}{c}\text { 30-Day } \\
\text { mortality rate }\end{array}$ \\
\hline Ascending/arch & 218 & $8(3.7 \%)^{*}$ & $21(9.6 \%)$ & $29(13.3 \%) \dagger$ \\
Descending & 63 & $8(12.7 \%)^{*}$ & $9(14.3 \%)$ & $17(27.0 \%) \dagger$ \\
Thoracoabdominal & 36 & $1(2.8 \%)^{*}$ & $2(5.6 \%)$ & $3(8.3 \%) \dagger$
\end{tabular}

The $P$ values shown are for the comparison between ascending/arch versus descending/thoracoabdominal.

${ }^{*}$ Statistically significant difference, $P=.047$.

$\ddagger$ Statistically significant difference: ascending/arch versus descending, $P=.010$; ascending/arch versus thoracoabdominal, $P=.020$; descending versus thoracoabdominal, $P=.001$.

to 0 in 22 cases. Although some of the improvement may be accounted for by gradually increasing surgical proficiency, later operative date (as a continuous variable) was not associated with decreased stroke rates (Table 6). Mortality during this time period decreased commensurately, to $7.4 \%$ (5/68) for ascending and arch operations and $13.6 \%$ (3/22) for descending and thoracoabdominal operations $(P=.082$ and $P=.384$, respectively); there were no deaths except in those patients presenting on an urgent basis.

\section{Discussion \\ Incidence}

Stroke was found in this review to be disturbingly frequent after aortic surgery (6.9\% for operations on the ascending aorta and $8.1 \%$ for those on the descending aorta). This incidence of stroke after aortic surgery is fully in line with prior reviews. ${ }^{1,6-8}$ Little information is known to us from the literature regarding stroke after descending aortic surgery for comparison with our data.

Occurrence of stroke is somewhat counterintuitive in operations on the descending aorta because the descending aorta, being downstream from the head vessels, might not be expected to be a significant source of air or particulate 
TABLE 10. Patients with stroke: Preoperative and intraoperative characteristic

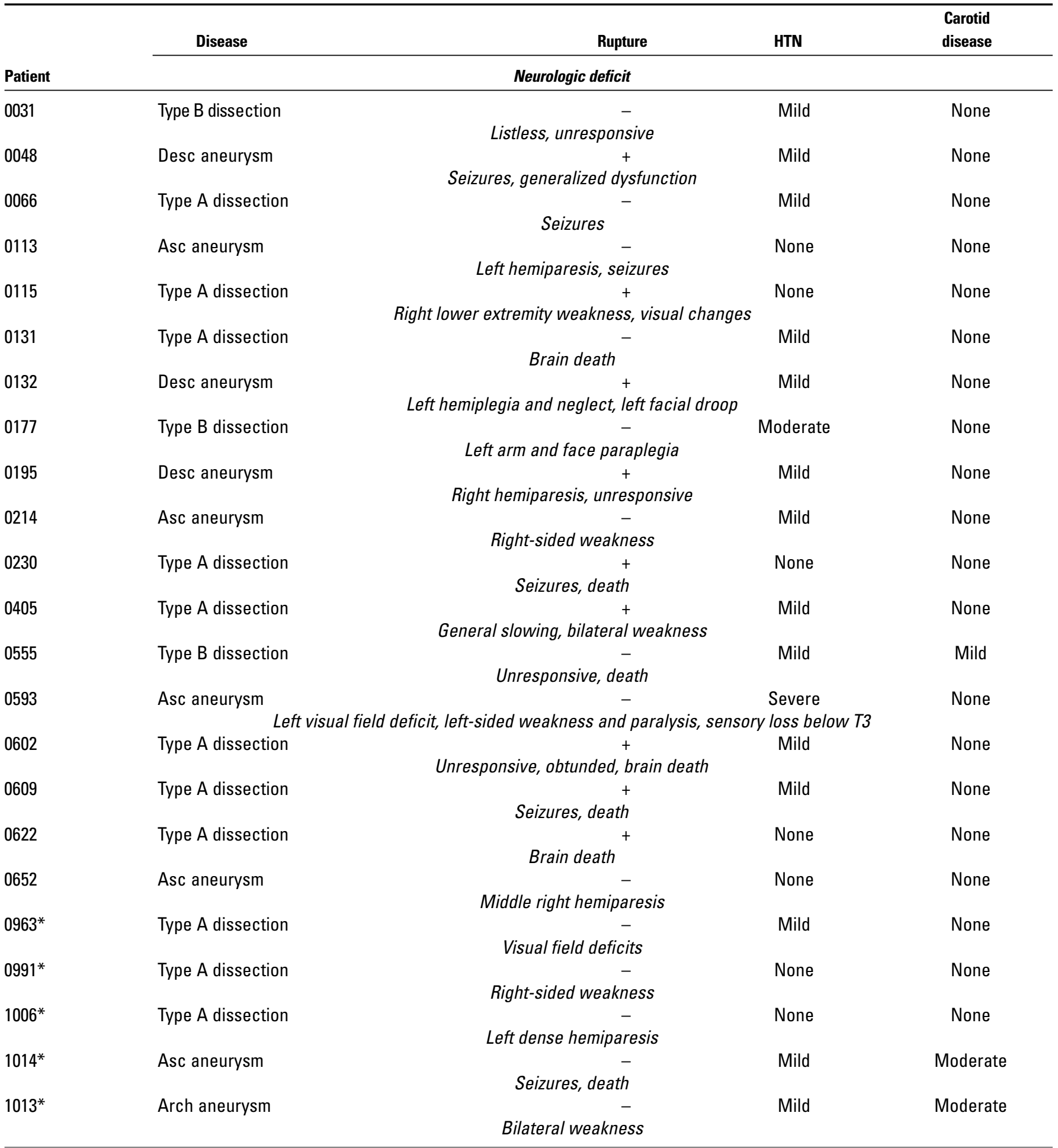

Desc, Descending; $A s c$, ascending; arch, aortic arch; $H T N$, hypertension; $N / A$, not available; $P O D$, postoperative day; $P V D$, peripheral vascular disease; $R A$, right atrium; $A$ o, aorta; $F A$, femoral artery; $F V$, femoral vein; $S C A$, subclavian artery; $D H C A$, deep hypothermic circulatory arrest; $C P B$, cardiopulmonary bypass; $A C A, M C A, P C A$, anterior, middle, and posterior cerebral arteries.

*Indicates patient operated on after the systematic use of antiembolic measures.

tStroke either embolic or due to hypoperfusion. 


\begin{tabular}{|c|c|c|c|}
\hline $\begin{array}{l}\text { Cannulation } \\
\text { site }\end{array}$ & $\begin{array}{c}\text { Cerebral } \\
\text { protection }\end{array}$ & $\begin{array}{c}\text { Stroke } \\
\text { etiology }\end{array}$ & $\begin{array}{l}\text { 30-Day } \\
\text { survival }\end{array}$ \\
\hline \multicolumn{4}{|c|}{ Imaging studies } \\
\hline None & N/A & Hemorrhagic & Death POD 9 \\
\hline \multicolumn{4}{|c|}{ Extensive left frontal intraparenchymal, diffuse intraventricular and subarachnoid hemorrhages } \\
\hline RA-Ao & DHCA & Embolic & Survived \\
\hline \multicolumn{4}{|c|}{ Bilateral cerebellar and frontal, right caudate, and parietal infarcts } \\
\hline \multicolumn{3}{|c|}{ Bilateral thalmic infarcts } & Survived \\
\hline RA-FA & DHCA & Embolic & Survived \\
\hline \multicolumn{4}{|c|}{ Evolving right cerebellar and frontal lobe infarcts without hemorrhage } \\
\hline RA-FA & CPB & Embolic & Survived \\
\hline \multicolumn{4}{|c|}{ Bilateral caudate and periventricular white matter infarcts } \\
\hline RA-FA & CPB & Ischemic & Death POD 5 \\
\hline \multicolumn{4}{|c|}{ Diffuse anoxic brain injury } \\
\hline Ao-FA & DHCA & Embolic & Survived \\
\hline \multicolumn{4}{|c|}{ Evolving right MCA infarcts } \\
\hline RA-FA & CPB & Embolic & Survived \\
\hline \multicolumn{4}{|c|}{ Early ischemia in the right MCA distribution } \\
\hline FV-FA & DHCA & Embolic & Survived \\
\hline \multicolumn{4}{|c|}{ Multiple bilateral infarcts } \\
\hline RA-FA & DHCA & Embolic & Death POD 20 \\
\hline \multicolumn{4}{|c|}{ Right frontal infarcts } \\
\hline RA-FA & DHCA & Embolic & Death POD 5 \\
\hline \multicolumn{4}{|c|}{ Extensive bilateral infarcts } \\
\hline RA-FA & DHCA & Ischemic & Survived \\
\hline \multicolumn{4}{|c|}{ Bilateral wedge-shaped parietal"watershed"infarcts } \\
\hline LA-FA & None & Hemorrhage & Death POD 1 \\
\hline \multicolumn{4}{|c|}{ Extensive, diffuse intraparenchymal, intraventricular, and subarachnoid hemorrhages } \\
\hline FV-FA & CPB & Embolic & Survived \\
\hline \multicolumn{4}{|c|}{ Subacute infarcts in the right parieto-occipital region and posterior portion of the right temporal lobe } \\
\hline RA-FA & DHCA & Unknown & Death POD 2 \\
\hline \multicolumn{4}{|c|}{ Imaging not performed } \\
\hline RA-FA & DHCA & Ischemic & Death POD 4 \\
\hline \multicolumn{4}{|c|}{ Bilateral cerebellar infarcts and ACA/MCA "watershed" and left PCA infarcts } \\
\hline RA-FA & CPB & Hemorrhage & Death POD 3 \\
\hline \multicolumn{4}{|c|}{ MCA infarctions with subsequent hemorrhagic conversion } \\
\hline RA-FA & DHCA & Embolic & Survived \\
\hline \multicolumn{4}{|c|}{ Right cerebellar infarct } \\
\hline None & N/A & Unknownt & Survived \\
\hline \multicolumn{4}{|c|}{ Occipital brain-stem/PCA infarct } \\
\hline RA-FA & DHCA & Embolic & Survived \\
\hline \multicolumn{4}{|c|}{ Left parietal infarct } \\
\hline RA-Ao & CPB & Embolic & Survived \\
\hline \multicolumn{4}{|c|}{ Right cerebellar infarcts } \\
\hline RA-SCA & DHCA & Embolic & Death POD 4 \\
\hline \multicolumn{4}{|c|}{ Multiple bilateral infarcts } \\
\hline RA-FA & DHCA & Embolic & Survived \\
\hline \multicolumn{4}{|c|}{ Multiple bilateral infarcts, diffuse, all lobes, left greater than right } \\
\hline
\end{tabular}


TABLE 11. Stroke rates

\begin{tabular}{lccccc}
\hline & Overall $(\mathbf{n}=\mathbf{3 1 7})$ & Ascending $(\mathbf{n}=\mathbf{2 1 8})$ & Descending $(\mathbf{n}=\mathbf{9 9})$ & Elective $(\mathbf{n}=\mathbf{2 0 6})$ & Emergency $(\mathbf{n}=\mathbf{9 7})$ \\
\hline $\begin{array}{l}\text { Without antiembolic } \\
\text { procedures }(\mathrm{n}=227)\end{array}$ & $18(7.9 \%)$ & $10(6.7 \%)$ & $8(10.4 \%)$ & $6(3.9 \%)$ & $12(16.2 \%)$ \\
$\begin{array}{l}\text { With antiembolic } \\
\quad \text { procedures }(\mathrm{n}=90)\end{array}$ & $5(5.6 \%)$ & $5(7.4 \%)$ & $0(0.0 \%)$ & $1(1.9 \%)$ & $4(17.4 \%)$ \\
$\begin{array}{l}\text { Overall } \\
P \text { values }\end{array}$ & $23(7.3 \%)$ & $15(6.9 \%)$ & $8(8.1 \%)$ & $7(3.4 \%)$ & $16(16.5 \%)$ \\
\hline
\end{tabular}

matter. This study found that stroke does indeed occur in descending aortic operations, in fact fully as frequently as in ascending aortic operations. We believe that manipulation of the aortic arch for proximal control in descending aortic operations is one factor. We believe that retrograde perfusion from below (femoral artery) is another factor producing stroke in descending aortic operations.

\section{Impact}

We have shown that stroke has a devastating impact on survival, producing death in the hospital in $28.3 \%$ of those affected and leaving only $28 \%$ alive at 2 years. Hospital morbidity was dramatically increased in stroke survivors, including intensive care unit stay, duration of intubation, and postoperative hospital stay. In addition to the usual concerns of death, bleeding, and paraplegia, surgeons must consider stroke heavily when deciding to operate on the thoracic aorta.

\section{Etiology}

In multivariable analysis, a number of risk factors were found to be predictive of stroke. These included a history of diabetes mellitus (possibly caused by chronic damage to the cerebral microvasculature), emergency surgery, the use and duration of hypothermic circulatory arrest, and the amount of transfused fresh frozen plasma (which may be a surrogate indicator of coagulopathy or intraoperative bleeding in these patients). Surprisingly, we found no correlation between the incidence of stroke and the location of either the proximal clamp in descending aortic surgery or the arterial cannulation site. The clinicoradiographic analysis revealed that the strokes in this study were predominantly (65.2\%) embolic in nature. An embolic origin would explain the failure to correlate with measurable preoperative characteristics in multivariable analysis, because embolism is a purely technical issue. Ergin and associates ${ }^{8}$ also concluded that permanent neurologic deficits were due to thromboembolic events and were not related to the type of cerebral protection used.

\section{Prevention}

Because intraoperative emboli are the overwhelming source of cerebrovascular accidents in this study, antiembolic precautions during the operative period become paramount.
The sobering impact of stroke on length and quality of life apparent in this review leads the surgeon to focus on potential modifications to operative technique to reduce the incidence of stroke in patients undergoing thoracic aortic surgery. Several practical recommendations arise as corollaries of the embolic etiologic mechanism of stroke.

In addition to the attention commonly given to minimize cardiopulmonary bypass time and deep hypothermic arrest interval, the surgeon must focus on antiembolic precautions. The following specific technical alterations in the conduct of surgery have been incorporated in our techniques and are recommended:

1. Mobilization. Even mobilization for operations on the severely diseased descending aorta can liberate debris to the cerebral vessels. Thus, extreme care is taken in manipulation of the aortic arch for proximal control in descending aortic operations.

2. Debridement. Severely diseased aortic cuffs are meticulously debrided of atheromatous debris before anastomosis and restoration of circulation, because remnants can easily be embolized to the head vessels.

3. Cannulation and perfusion. Transesophageal echocardiography can map the location and severity of aortic atheromatous disease and direct the surgeon toward different cannulation and perfusion approaches. ${ }^{9-11}$ In patients with severe, mobile atheroma of the descending aorta, the surgeon may be presented with unfavorable conditions for retrograde femoral perfusion. In these cases, alternative perfusion sites, for example, the subclavian or axillary arteries, may prove effective at preventing embolization of debris, especially inasmuch as these sites provide "antegrade" flow. Retrograde flow may be more likely to lift the "shingle-like" atheromas molded by years of natural antegrade flow.

4. Carbon dioxide flooding. Our institution uses carbon dioxide flooding of the operative field. Because carbon dioxide is heavier than atmospheric air, it will displace this air. Theoretically, any gas embolized from the field will be carbon dioxide, which will more easily dissolve once in the cerebral circulation and pose less of a risk to the patient. This modality has an excellent therapeutic ratio: essentially no cost and very significant potential benefits.

5. Order of clamping. We recommend application of the proximal clamp (in descending aortic surgery) before the 
institution of femoral perfusion to prevent any disrupted atheromatous debris from reaching the cerebral circulation. Similarly, we discontinue perfusion before release of the proximal clamp.

6. Avoidance of the left atrium in atrial fibrillation. In patients with atrial fibrillation, we avoid cannulating the left atrial appendage for left atrial-femoral bypass for descending aortic surgery, for fear of liberating debris from the appendage by the cannulation process. Femoro-femoral bypass or deep hypothermic arrest may be preferable techniques.

The decrease in stroke rate from $8.1 \%$ to $0.0 \%$ for operations on the descending aorta in the time period after these observations were made and systematic antiembolic measures instituted suggests a beneficial impact of these measures. Stroke is an important cause of mortality, and mortality has also decreased during the postimplementation period. These outcomes at our institution have already shown moderate to strong beneficial trends in some parameters after implementation of a rigorous antiembolic regimen. To demonstrate precise statistical significances from the implementation of antiembolic measures would require huge patient numbers; we think the demonstrated trends are suggestive of a beneficial impact.

We conclude that potential for postoperative strokes is significant after surgery of the thoracic aorta and needs careful inclusion in weighing the pros and cons of surgical intervention for a particular patient. The risk of stroke is about $7 \%$. Stroke is often lethal $(28.3 \%)$, accounting for one quarter of all deaths from thoracic aortic surgery. Among those patients who survive to be discharged from the hospital after a stroke, only $28 \%$ will be alive 2 years after thoracic aortic resection. The majority of strokes are embolic-technically related. Specific technical correlates for prevention present themselves once the embolic origin is appreciated. Application of these technical correlates may help to decrease the incidence of stroke, a devastating complication of thoracic aortic surgery.

\section{References}

1. Livesay JJ, Cooley DA, Reul GJ, Walker WE, Frazier OH, Duncan JM, et al. Resection of aortic arch aneurysms: a comparison of hypothermic techniques in 60 patients. Ann Thorac Surg. 1983;36:19-28.

2. Okita Y, Takamoto S, Ando M, Morota T, Matsukawa R, Kawashima Y. Mortality and cerebral outcome in patients who underwent aortic arch operations using deep hypothermic circulatory arrest with retrograde cerebral perfusion: no relation of early death, stroke, and delirium to the duration of circulatory arrest. J Thorac Cardiovasc Surg. 1998;129-38.

3. Raskin SA, Fuselier VW, Reeves-Viets JL, Coselli JS. Deep hypothermic circulatory arrest with and without retrograde cerebral perfusion. Int Anesth Clinics. 1996;34:177-93.

4. Bachet J, Guilmet D, Goudot B, Dreyfus GD, Delentdecker P, Brodaty $\mathrm{D}$, et al. Antegrade cerebral perfusion with cold blood: a 13-year experience. Ann Thorac Surg. 1999;67:1874-8.

5. Rutherford RB, Flanigan DP, Gupta SK, Johnston KW, Karmody A, Whittemore AD, et al. Suggested standards for reports dealing with lower extremity ischemia. J Vasc Surg. 1986;4:80-94.

6. Svensson LG, Crawford ES, Hess KR, Coselli JS, Raskin S, Shenaq SA, et al. Deep hypothermia with circulatory arrest: determinants of stroke and early mortality in 656 patients. J Thorac Cardiovasc Surg. 1993;106:19-31.

7. Bavaria JE, Woo J, Hall A, Wahl PM, Acker MA, Gardner TJ. Circulatory management with retrograde cerebral perfusion for acute type A aortic dissection. Circulation. 1996;94(Suppl):II-173-6.

8. Ergin MA, Galla JD, Lansman SL, Quintana C, Bodian C, Griepp RB. Hypothermic circulatory arrest in operations on the thoracic aorta. $J$ Thorac Cardiovasc Surg. 1994;107:788-99.

9. Amarenco P, Cohen A, Tzourio C, Bertrand B, Hommel M, Besson G, et al. Atherosclerotic disease of the aortic arch and the risk of ischemic stroke. N Engl J Med. 1994;331:1474-9.

10. Ribakove GH, Katz ES, Galloway AC, Grossi UA, Esposito RA, Baumann G, et al. Surgical implications of transesophageal echocardiography to grade the atheromatous aortic arch. Ann Thorac Surg. 1992;53:758-63.

11. Wareing TH, Davila-Roman VG, Barzilai B, Murphy SF, Kouchoukos NT. Management of the severely atherosclerotic ascending aorta during cardiac operations. J Thorac Cardiovasc Surg. 1992;103:453-62. 